\section{Año 2019-2020. urtea N. ${ }^{\circ} 31-32 . z k$.}

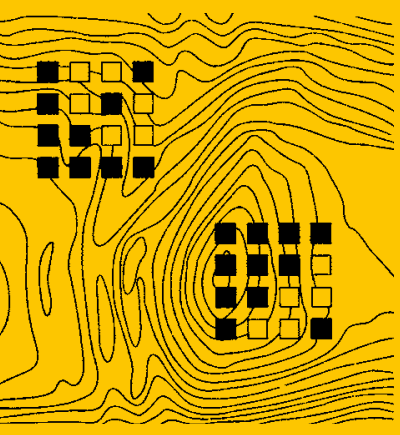

\section{TRABAJOS DE ARQUEOLOGÍA NAVARRA}

SEPARATA

\title{
Trabajos arqueológicos en el yacimiento de Irulegi (Valle de Aranguren), 2019-2020
}

Mattin Aiestaran de la Sotilla, Daniel Ruiz González, Eneko Iriarte Avilés, Jesús Sesma Sesma, Jesús García Gazólaz, Jose Antonio Mujika Alustiza, Juantxo Agirre Mauleon

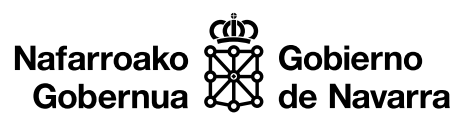




\section{Trabajos arqueológicos en el yacimiento de Irulegi (Valle de Aranguren), 2019-2020}

Irulegiko aztarnategiko (Aranguren ibarra) jarduera arkeologikoak 2019-2020

Archaeological works at the site of Irulegi (Aranguren valley), 2019-2020

Mattin Aiestaran de la Sotilla

Sociedad de Ciencias Aranzadi. UPV/EHU

maiestaran@aranzadi.eus

Daniel Ruiz González

Sociedad de Ciencias Aranzadi. UPV/EHU

Eneko Iriarte Avilés

Universidad de Burgos

Jesús Sesma Sesma

Gobierno de Navarra

Jesús García Gazólaz

Gobierno de Navarra

Jose Antonio Mujika Alustiza

Universidad del País Vasco/Euskal Herriko Unibertsitatea (UPV/EHU)

Juantxo Agirre Mauleon

Sociedad de Ciencias Aranzadi

DOI: https://doi.org/10.35462/TAN31-32.9

Agradecemos al Ayuntamiento del Valle de Aranguren y a los vecinos del valle por el apoyo económico y la colaboración ejemplar, así como la asistencia y ayuda del Gobierno de Navarra. Damos las gracias también a la redacción de la revista por las aportaciones realizadas para mejorar el texto final.

Este artículo se enmarca en una tesis doctoral becada con la ayuda de la UPV/EHU PIF 2019, participado por el Grupo de Investigación en Prehistoria IT-1223-19. 


\section{RESUMEN}

Entre los años 2019 y 2020 un equipo de la Sociedad de Ciencias Aranzadi ha llevado a cabo una serie de trabajos arqueológicos en el poblado de la Edad del Hierro del yacimiento de Irulegi, en el municipio de Laquidáin (valle de Aranguren). Durante varias campañas se han realizado prospecciones visuales, prospecciones geofísicas, sondeos geoarqueológicos y excavaciones arqueológicas, tanto en forma de catas como en área abierta, que han permitido empezar a conocer esta fase del yacimiento de la prehistoria reciente.

Palabras clave: Irulegi; valle de Aranguren; poblado de la Edad del Hierro; arqueología.

\section{LABURPENA}

2019 eta 2020 urteetan Aranzadi Zientzia Elkarteko talde batek Lakidaingo herrian (Aranguren Ibarra) dagoen Irulegiko Burdin Aroko herrixkan zenbait jarduera arkeologiko burutu ditu. Kanpaina hauetan miaketak, miaketa geofisikoak, zundaketa geoarkeologikoak eta indusketa arkeologikoak, bai miaketa-zulo eta baita area zabalean planteatuak, egin dira, aztarnategi arkeologiko honetako historiaurre berantiarreko fase honen ikerketa hasiz.

Gako hitzak: Irulegi; Aranguren ibarra; Burdin Aroko herrixka; arkeologia.

\section{ABSTRACT}

Between 2019 and 2020, a team from the Aranzadi Science Society carried out a series of archaeological works in the Iron Age town of the Irulegi site, in the municipality of Laquidáin (valle de Aranguren). During several campaigns, visual surveys, geophysical surveys, geoarchaeological surveys and archaeological excavations have been carried out, both in the form of trenches and in an open area, which have allowed us to begin to know this phase of this site.

Keywords: Irulegi; Aranguren Valley; Iron Age hillfort; Archaeology. 
1. LOCALIZACIÓN Y ANTECEDENTES DE LA INTERVENCIÓN. 2. TRABAJOS REALIZADOS. 3. CONCLUSIONES. 4. BIBLIOGRAFÍA.

\section{LOCALIZACIÓN Y ANTECEDENTES DE LA INTERVENCIÓN}

La documentación escrita de época medieval custodiada en el Archivo General de Navarra recoge numerosas menciones en torno al castillo de Irulegi, sobre las obras realizadas y la manutención de su guarnición. Además, en la historiografía, las crónicas y las monografías históricas se ha mencionado una y otra vez su existencia. Entre estos estudios históricos debemos destacar los trabajos de Juan José Martinena $(1994,2017)$ y una monografía de Iñaki Sagredo (2006). En cuanto a los restos arqueológicos, se han de mencionar los estudios y prospecciones realizados por Amparo Castiella y otros investigadores (Castiella et al., 1999), que supusieron un primer acercamiento científico al estudio de los hábitats protohistóricos de la Cuenca de Pamplona, dando a conocer el yacimiento arqueológico del monte Irulegi.

Atendiendo a todo ello, en el año 2007 el Ayuntamiento del Valle de Aranguren promovió la realización de excavaciones arqueológicas, que durante los diez años siguientes se centraron prioritariamente en la investigación y consolidación del castillo medieval. Las actuaciones se han realizado gracias a una partida económica que el Ayuntamiento reservaba anualmente de sus propios recursos, y también gracias al voluntariado del vecindario del valle y de alumnos universitarios de Arqueología e Historia que han venido realizado prácticas de campo.

Tras diez años de trabajos, los restos del castillo medieval están musealizados y son muy numerosas las visitas que reciben. Se ha convertido en un espacio referente para el ocio verde y cultural en Iruñerria-Cuenca de Pamplona. 
En cuanto al poblado de la Edad del Hierro, se han de mencionar la tesis doctoral y publicaciones de Javier Armendáriz (2005, 2008, 2009), que, con una importante mención al yacimiento de Irulegi, supusieron un hito en la historiografía navarra.

Sin embargo, hay que subrayar las investigaciones que la Sociedad de Ciencias Aranzadi ha llevado a cabo desde el año 2007, que, habiéndose centrado en el castillo medieval (Buces et al., 2013), han tenido como objetivo delimitar y establecer las distintas áreas del yacimiento. Para ello, en paralelo, se realizó un sondeo en la zona del poblado en 2007. En dicho sondeo se hallaron tres niveles claramente diferenciados con restos de estructuras y materiales asociados a tipologías de la Edad del Hierro. Asimismo, se han de mencionar los trabajos de prospección geofísica de SOT y S. C. Aranzadi (2012). Las prospecciones magnéticas y eléctricas evidenciaron la buena conservación de la planta del poblado.

\section{TRABAJOS REALIZADOS}

En el sondeo realizado en 2018 y proseguido en 2019, se documentaron los restos de una estructura posiblemente habitacional de la Edad del Hierro. En las prospecciones geofísicas realizadas en 2018 se observaba un contraste magnético que fue interpretado como de combustión. Durante la excavación se han registrado tres grandes fases cronológicas diferenciadas mediante la estratigrafía, las tipologías cerámicas y los materiales arqueológicos. Las dataciones relativas y absolutas que se han ido obteniendo gracias a los registros excavados ponen de manifiesto que este es un yacimiento con una diacronía que engloba amplias fases cronológicas que pueden tener, o no, continuidad.

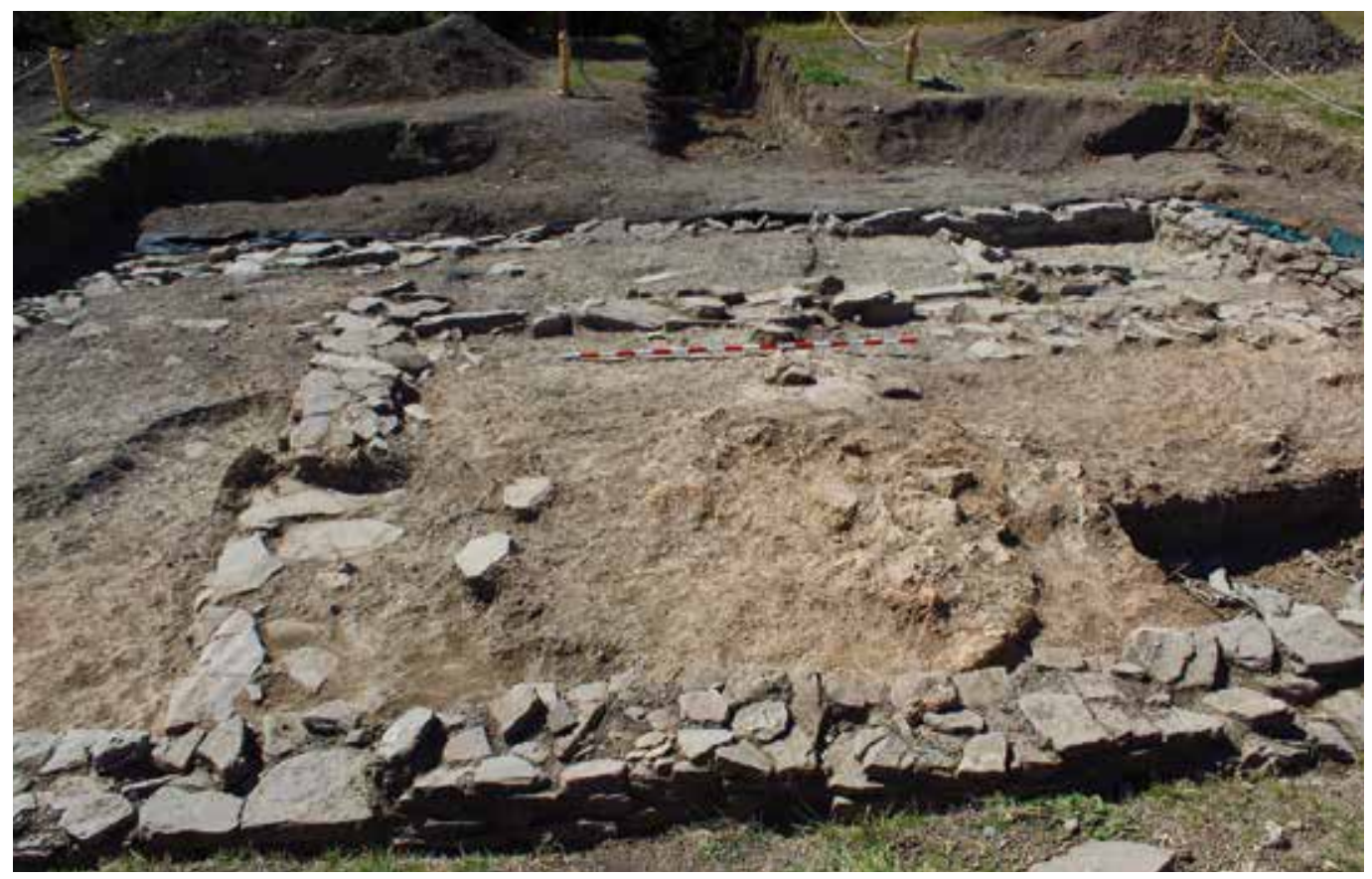

Figura 1. Imagen de la excavación arqueológica. Mattin Aiestaran.

Trabajos de Arqueología Navarra (TAN), 31-32, 2019-2020, 223-229 ISSN: 0211-5174 ISSN-e: 2530-5816 ISSN-L: 0211-5174 
Estas incipientes hipótesis fueron corroboradas por los resultados de la excavación en área abierta de 2020, la cual tenía como objetivo la excavación de la estancia que se comenzó a conocer en 2018 y 2019.

La constatación de diversas fases de edificios datados en la Edad del Hierro Avanzada y Final aporta datos novedosos acerca del urbanismo de este tipo de poblados en el prepirineo y sobre todo en la Cuenca de Pamplona-Iruñerria. Su gran tamaño y morfología cuadrangular apuntan hacia una vivienda con influencia romana. El análisis preliminar de la cultura material recuperada señala la misma hipótesis, ya que aparecen bajo y en el nivel de derrumbe por destrucción intencionada restos bélicos de adscripción romana y una vasija de tipología cerámica de importación campaniense de la misma época, acompañado de cerámica de tipo celtibérico. Por lo tanto, se ha podido concretar la posible interpretación de varios aspectos referentes al urbanismo de la fase final, algunas de sus características y su violento e intencionado final. Todo ello hace de este yacimiento una pieza clave para entender los procesos conflictivos e inestabilidad con la llegada de la romanización.

Por otra parte, en la campaña de 2019 se realizaron varios sondeos geoarqueológicos para continuar documentando y validando las interpretaciones geofísicas. Dichos sondeos han permitido conocer la estratigrafía de la zona superior del yacimiento (la campa bajo el castillo medieval), así como validar las interpretaciones de las prospecciones geofísicas. Asimismo, durante la realización de dichos sondeos se han recogido muestras que permitirán la realización de análisis sedimentológicos, palinológicos, etc.

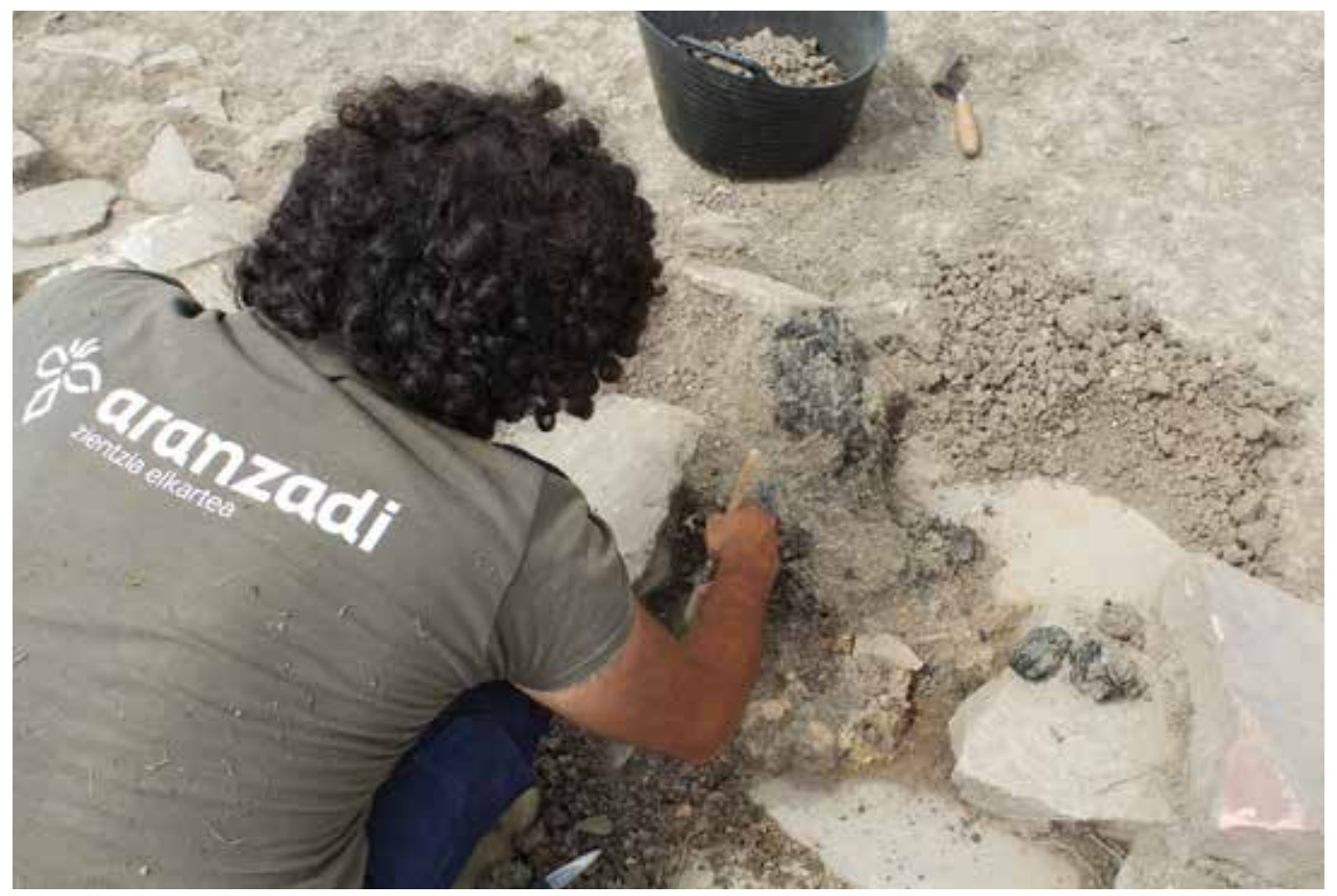

Figura 2. Imagen del proceso de excavación. Mattin Aiestaran. 
En 2019 también se realizaron varias catas arqueológicas en las murallas perimetrales e internas. En ellas hemos podido documentar y poner de manifiesto que las novedosas interpretaciones de las irregularidades del terreno identificadas en las prospecciones realizadas en la campaña anterior son acertadas, y que estas responden a defensas perimetrales de importantes dimensiones. De todos modos, se pudo constatar que la conservación de las defensas no es la misma en todas las zonas.

\section{CONCLUSIONES}

Con los trabajos realizados hasta ahora estimamos que la totalidad del poblado (incluyendo: núcleo urbano, recintos de función ganadera/agraria, murallas, etc.), podría tener una extensión máxima cercana a las diez hectáreas, aunque se desconozca la naturaleza de la campa aterrazada del sur de ocho hectáreas aproximadas. Sin embargo, esta zona estaba fuertemente defendida por defensas artificiales de considerables dimensiones.

A esto último se le suma la excelente conservación de los restos hallados. La planificación de la trama urbana que muestran las distintas construcciones, con fases superpuestas y distintas reparaciones o modificaciones, abre la posibilidad de investigar a fondo las sociedades que habitaban este tipo de hábitats concentrados y fortificados. A pesar de contar con una realidad urbana aparentemente compleja, Irulegi no llega a tener las grandes extensiones de los oppida de otras zonas de la península ibérica o el valle del Ebro, pero sí una extensión y desarrollo urbano importante comparado con los poblados de la zona de alrededor. Eso mismo lo hace interesante de cara a entender los patrones de asentamiento de la Navarra Media o la Navarra de las cuencas prepirenaicas.

Así pues, en el monte Irulegi se sitúa un yacimiento arqueológico de primer orden en los ámbitos de la investigación científica de la historia de Navarra y en el del disfrute del patrimonio cultural. En lo que respecta a la investigación histórica de nuestro pasado, este yacimiento es fundamental para conocer la evolución del poblamiento de la Edad de Hierro en Iruñerria-Cuenca de Pamplona y los procesos de romanización.

\section{BIBLIOGRAFÍA}

Armendáriz Martija, J. (2005). Propuesta de identificación del campamento de invierno de Pompeyo en territorio vascón. Trabajos de Arqueología Navarra, 18, 41-64.

Armendáriz Martija, J. (2008). De aldeas a ciudades. El poblamiento durante el primer milenio a. C. en Navarra. Pamplona: Gobierno de Navarra.

Armendáriz Martija, J. (2009). La ordenación del territorio vascón en la Edad del Hierro. En J. Andreu (coord), Los vascones de las fuentes antiguas (pp. 303338). Barcelona: Edicions Universitat de Barcelona.

Buces, J., Moraza, A., Agirre, J., Pescador, A. \& Legorburu, M. (2013). Un enclave estratégico en la Cuenca de Pamplona: el castillo medieval de Irulegi 
(Lakidain, Navarra). Balance de los trabajos arqueológicos (2007-2012). Trabajos de Arqueología Navarra, 25, 143-167.

Castiella, A., Sesma, J., García, M. L., García, J., Prieto, J. J., Faro, J. A. \& García, D. (1999). Poblamiento y territorialidad en la Cuenca de Pamplona: una visión arqueológica. Cuadernos de Arqueología de la Universidad de Navarra, 7.

García, E., Sala, R., Tamba, R. \& Buces, J. (2012). Prospección geofísica multisistema en el yacimiento arqueológico de Irulegi (Lakidain, Nafarroa). En $7 .^{a}$ Asamblea hispano-portuguesa de Geodesia y Geofísica. Donostia.

Martinena, J. J. (1994). Castillos reales de Navarra (siglos XIII-XVI). Pamplona: Gobierno de Navarra.

Martinena, J. J. (2017). El castillo de Irulegui. Zangotzarra, 21, 40-65.

Sagredo, I. (2006). El castillo de Irulegi. Ayuntamiento del Valle de Aranguren. 